(Aus der Königl. Universitäts-Frauenklinik zu Königsberg i. Pr. Director: Prof. Dr. G. Winter.)

\title{
Ueber die Ausscheidung der Chloride in der Schwangerschaft, speciell bei Nephritis gravidarum.
}

Von

Privatdocent Dr. W. Zangemeister, Königsberg i. Pr.

Die Frage der Kochsalzausscheidung in der Schwangerschaft hat dadurch in letzter Zeit ein praktisches Interesse gewonnen, dass Cramer ${ }^{1}$ ) den Versuch gemacht hat, den Graviditätshydrops mit Kochsalzentziehung zu behandeln, und zwar im Hinblick auf die Empfehlung dieses Vorgehens für nephritische Oedeme ${ }^{2}$ ) im Allgemeinen.

Cramer hat, wie er neuerdings wieder versichert ${ }^{3}$ ), günstige Resultate erzielt, das Verfahren hat sich "glänzend bewährt". Es liegt mir durchaus fern, die guten Erfolge Cramer's bezweifeln zu wollen. Praktische Erfahrungen gehen über alle theoretischen Erwägungen. Aber da ich in der Lage bin, auf Grund früherer, zum Theil sehr eingehender Arbeiten die Ausscheidungsverhältnisse der normalen und kranken Niere in der Schwangerschaft zu beurtheilen, so kann ich den Verdacht nicht unterdrücken, dass Cramer's günstige Resultate auf andere Momente als auf die Kochsalzentziehung zurückzuführen sind. Jeder von. uns weiss, wie schnell derartige Oedeme - zumal wenn sie, wie die Cramer's, nicht mit Albuminurie verbunden sind - oft zurückgehen. Bettruhe allein wirkt meist prompt; ja schon die geregeltereLebensweise, welche

1) Monatsschr. f. Geb. u. Gyn. Bd. XXIII. S. 437.

2) Ich verweise bier z. B. auf Strauss, Ther. d. Gegenwart. Mai 1903; Kövesi u. Roth-Schulz, Berl. klin. Wochenschr. 1904. No. 24-26; Halpern, Festschr. f. Salkowski. 1904.

3) Münch. med. Wochenschr. 1907. S. 2639. 
eine Patientin einhält, wenn sie erst einmal unter ärztlicher Beobachtung in irgend einer Form steht, wird - auch ohne jede zweckdienliche Therapie - einen günstigen Einfluss haben können. Ich will damit nur sagen, dass gute Erfolge nur mit äusserster Vorsicht zu beurtheilen sind und an einem grossen Material bestätigt werden müssen, am besten derart, dass abwechselnd eine Kranke mit Kochsalzentziehung behandelt wird, die nächste nicht u. s. f. Es dürfte sich verlohnen, in dieser Weise den Werth des Verfahrens zu prüfen.

Cramer hat es nicht unternommen, seine Resultate experimentell zu stützen; er hat auch nicht den Versuch gemacht, an der Hand der in unserer Literatur niedergelegten Untersuchungen Anderer eine Stütze für seine Ansicht zu finden, wiewohl genügendes Material hierzu vorlag. Der Autor beschränkte sich eben ganz auf die praktische Seite der Frage.

Auffallender ist es, dass Birnbaum ${ }^{1}$ ), welcher der Frage theoretisch nachgeht, die Literatur der letzten Jahrgänge nicht kennt. Birnbaum hat sich (l. c., S. 656) drei Fragen vorgelegt, deren experimentelle Beantwortung die Wirkungsweise der Kochsalzentziehung aufklären soll. Die Fragen 1 und 3 hätte der Autor ohne Weiteres aus den Ergebnissen meiner diesbezüglichen Arbeiten 2) beantworten können, und zwar wesentlich präciser, als es nach seinen Untersuchungen - über die Chloridausscheidung in der Schwangerschaft und bei Hydrops und bei Nephritis gravid. möglich ist. Um so erfreulicher ist es, dass Birnbaum - offenbar also völlig unabhängig - so weit seine Untersuchungen reichen, zu den gleichen Ergebnissen gekommen ist wie ich.

Die von Birnbaum angewandte Methode der Chloridbestimmung ist die nach Volhard-Arnold-Salkowski; ich habe diese Methode seiner Zeit ${ }^{3}$ ) auf Grund zahlreicher Versuche für den hier in Frage stehenden Zweek dahin verbessert bezw. vereinfacht, dass ein und dasselbe (sebr einfache und zuverlässige) Titrationsverfahren für eiweissfreien, eiweisshaltigen Urin und Blutserum anwendbar ist.

Ich werde versuchen, an der Hand des insgesammt jetzt vorliegenden Materials die Frage zu entscheiden, ob in der normalen Schwangerschaft, ferner bei Nephritis gravid. und Eklampsie eine Kochsalzretention besteht. Ich kann mich dabei kurz fassen,

1) Dieses Arch. Bd. 83. S. 653 .

2) Zeitschr. f. Geb. u. Gyn. Bd. 49. S. 92 u. Bd. 50. S. 385.

3) Zeitschr. f. Geb. u. Gyn. 49. S, 96 Anm. 
da ich diese Fragen in meinen Arbeiten bereits mehrfach berührt habe.

Die Harnmenge ist am Ende der Schwangerschaft gegen die Norm etwas erhöht1). Das beweisen ausser den Untersuchungen von Winckel's ${ }^{2}$ ) und Kehrer's ${ }^{3}$ ) die meinigen ${ }^{4}$ ). Die absolute Menge hängt von der Aussentemperatur ab; daher bestehen gewisse Differenzen in den Angaben.

\begin{tabular}{|c|c|c|c|}
\hline Kehrer & (39 & 7 & ): 1792 \\
\hline Zangemeister & $(22$ & $n$ & ): 1670 \\
\hline$n$ & $(13$ & $n$ & ): 1510 \\
\hline Birnbaum & $(16$ & $n$ & ): 1465 \\
\hline
\end{tabular}

In der Norm beträgt die 24 stündige Harnmenge:

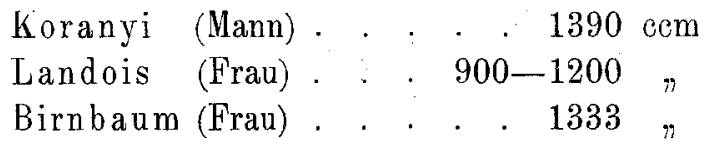

Die in 24 Stunden ausgeschiedene Kochsalzmenge beträgt bei Schwangeren: von Winckel (l. c.) . . . . . . . 15,805) Zangemeister (l. c., S. 409 u. 435) . . . 12,85 (8,1-17,5) Birnbaum (l. c., S. 661) . . . . . . 12,82 (9,5-16,7)

bei Nichtschwangeren:

Spaeth und Neubaur-Vogel (Mann) . . 15,0 (9,0-25,0) Birnbaum (Frau) . . . . . . . . . 13,2 (10,2-15,0)

Wenn ich demnach (Zeitschr. f. Geb. Bd. 50. S. 411 u. 437) gesagt habe, die 24 stündige Chloridmenge sei bei Schwangeren im Ganzen der Norm (i. e. nicht gravide Frau) entsprechend, vielleicht etwas verringert, so kann ich diese These heute voll und ganz wiederholen.

Wenn Birnbaum allein auf Grund der Thatsache, dass die Chloridausscheidung in der Schwangerschaft nicht wesentlich gegenüber der Norm herabgesetzt ist, bereits den Schluss zieht, dass eine Kochsal.

1) Birnbaum (l. c., S. 662) giebt irrthümlicher Weise an, ich hätte eine Verminderung der Urinmenge festgestellt.

2) v. Winckel, Handb. d. Geb. Bd. I. S. 376.

3) P. Müller, Handb. d. Geb. Bd. I. S. 565.

4) Hegar's Beitr. Bd. V. S. 317. - Dieses Arch. Bd. 66. S. 414. Zeitschr. f. Geb. u. Gyn. Bd. 50. S. 409, 410, 435.

5) Die von r. Winckel benutzte Mohr'sche Methode ergiebt bekanntlich zu hohe Werthe. 
retention bei gesunden Schwangeren nicht vorhanden sei, so ist das übereilt. Denn: relativ ungenügend könnte die Chloridausscheidung dennoch sein, da weder die Kochsalzaufnahme in der Schwangerschaft noch der Chloriảstoffwechsel des Fötus mit in Rechnung gezogen ist.

Ein wesentlich klareres Bild erhalten wir über die Ausscheidungsverhältnisse der Schwangerenniere, wenn wir die Gesammtsalze mit in Betracht ziehen, wie dies nach dem Vorgange von Koranyi durch die Gefrierpunktsbestimmung geschehen kann.

Nach Abzug der Chloride von den Gesammtsalzen (ebenfalls auf $\mathrm{NaCl}$ berechnet) erhalten wir die Summe aller Nichtchloride, deren Ausscheidungsverhältnisse gänzlich andere sind. $\mathrm{Zu}$ ihnen gehören einmal diejenigen Stoffe, welche als Abfallsproducte anzusehen sind (Stickstoffgruppe), ferner diejenigen Salze, welche zum Aufbau des Fötus dienen und im fötalen Kreislauf zu diesem Zwecke zurückgehalten, verbraucht werden (Phosphate, Carbonate etc.).

Diese Nichtchloride sind im Schwangerenharn ganz beträchtlich verringert ${ }^{1}$ ): $9,63 \mathrm{~g}$ (als $\mathrm{NaCl}$ berechnet) gegenüber 25,75 in der Norm (Koranyi), wenngleich letztere Zahl bei der Frau wohl etwas kleiner ist.

Trotz dieser Verringerung der Achloridausscheidung handelt es sich aber bestimmt nicht um eine, durch Insufficienz der Ausscheidungsorgane bedingte Retention der Nichtchloride; deın einmal sehen wir, dass unter Umständen die Schwangerenniere ganz erheblich höhere Mengen von Achloriden auszuscheiden fähig ist, und zweitens widerspräche jener Annahme die Blutzusammensetzung. Genaue Analysen des Schwangerenblutes ergaben nämlich, dass auch im Blut die Nichtebloride relativ spärlich vertreten sind ${ }^{2}$ ), sodass wir zu der Annahme gezwungen sind, dass es eben jene veränderte Blutzusammensetzung ist, welche die Achloridarmuth des Harns in der Schwangerschaft bedingt. Wie ich oben andeutete, bringe ich diese Thatsache in Zusammenhang mit dem Verbrauch von Salzen seitens der wachsenden Frucht. Die Blutanalysen baben aber zugleich unzweideutig gezeigt, dass das Sohwangerenblut relativ reich an Chloriden ist ${ }^{3}$ ); es enthielt nämlich das Serum Schwangerer $6,5 \mathrm{pM}$. $\mathrm{NaCl}(6,3-6,7)$ gegenüber $6,0-6,2$ bei nicht schwangeren

1) Zangemeister, Zeitschr. f. Geb. u. Gyn. 50. S. 437.

2) Zangemeister, Zeitsohr. f. Geb. u. Gyn. 49. S. 100 u. 50. S. 437. Vergl. auch die Tabelle Bd. 50. S. 458.

3) Zeitschr. f. Geb. u. Gyn. 50. S. 98 u. 99. 
Frauen. Während der Geburt und im Wochenbett ging dieser erhöhte Chloridgehalt wieder zurück. Meine Analysen habe ich damals mehrfach wiederholt, stets controlirt; ihre Fehlergrenzen bestimmt, sodass ein Zweifel an dem erwähnten Ergebniss nicht bestehen kann.

Birnbaum (l. c. p. 662) hat ebenfalls den Chloridgehalt des Blutserums untersucht, und zwar an 2 Schwangeren und einer Nichtschwangeren: sein Resultat ist (die Ursache kann nur in einem Berechnungsfehler liegen!) ein unmögliches: Chlorgehalt des Blutserums

$$
\begin{aligned}
& \text { Schwangerer: 1) } 0,1740 \mathrm{pCt}=0,287 \mathrm{pCt} . \mathrm{NaCl} \\
& \text { 2) } 0,1775 \quad n=0,293 \quad n " \\
& \text { Nichtschwangerer: 3) } 0,1733, "=0,286 " n
\end{aligned}
$$

Es besteht also während der Schwangerschaft thatsächlich eine Chlorid-"retention" im Organismus; denn da einerseits die $\mathrm{NaCl}$-Concentration eine höhere, und andererseits die gesammte Blutmenge in der Gravidität erhöht ist, beherbergt allein schon das Blut mehr $\mathrm{NaCl}$ bezw. Chloride, als in der Norm.

Handelt es sich aber bei dieser Chloridretention um eine durch Niereninsufficienz bedingte Anhäufung eines vielleicht schädlichen Salzes? - Keineswegs!

In seinem Lehrbuch der Pathologie des Stoffwechsels hat schon von Noorden ${ }^{1}$ ) darauf hingewiesen, dass unter den harnfähigen Stoffen das $\mathrm{NaCl}$ selbst bei Nephritis leicht ausgeschieden wird.

Hohr (Zeitschr. f. klin. Med. 51) fand ebenfalls, dass die Ausscheidung des Kochsalzes bei acuter und chronischer Nephritis unbehindert ist; nur bei acuter Nephritis mit Oedemen trat Retention von Kochsalz auf. Diese Retention ist aber, wie unten noch erörtert wird, wahrscheinlich auch dort ein compensatorischer Vorgang, eine Folge der Wasserretention.

Zu gleichem Ergebniss kamen die experimentellen Untersuchungen Richter's (Berl. klin. Wochenschr. 1905. No. 14); auch er fand, dass das Kochsalz bei der Entstehung von Wassersucht nicht die ihm vielfach zugeschriebene Rolle spielt.

Auch Stölzner bestreitet, dass das Zustandekommen nephritischer Oedeme auf primäre Kochsalzretention zurückzuführen sei (Münch. med. Wochenschr. 1905. S. 2000).

Wir wissen ferner - und ich habe dies auch experimentell nachgewiesen -, dass die Schwangerenniere, wenn es darauf ankommt, ganz enorme Mengen von Chloriden auszuscheiden vermag. Selbst die schwerkranke Schwangerenniere (Nephritis gravid., Eklampsie) kann längere Zeit hindurch einen an Chloriden hoch concentrirten Harn liefern.

1) Berlin, Hirschwald 1893. S. $366 \mathrm{ff}$. 
Auch die Arbeiten von Magnus ${ }^{1}$ ). müssen bei der Beurtheilung der Frage herangezogen werden. Magnus zeigte, dass überschüssige Kochsalzmengen sofort aus dem Blut, theils in den Harn, theils in die Gewebe überführt werden; ferner, dass bei Zufuhr grosser Mengen $\mathrm{NaCl}$ dem Körper Wasser entzogen wird, welches verbraucht wird, um das eingeführte $\mathrm{NaCl}$ gehörig zu verdünnen und dann mit dem Harn fortzuspülen. Umgekehrt wird das $\mathrm{NaCl}$ des Organismus verbraucht, wenn Wasser im Ueberschuss zugeführt wird. Das $\mathrm{NaCl}$, als das überall im Körper in in grösseren Mengen disponible Salz, soll das eingeführte Wasser dem Organismus möglichst isotonisch machen. Wasserzufuhr entzieht also dem Körper Kochsalz.

Die Chloride haben somit die Aufgabe, helfend einzugreifen, um die Isotonie des Blutes za wahren, sie sind ungiftige, leicht lösliche Salze, die ihrerseits andere, schwer lösliche Salze in Lösung halten, und die auch unter den ungünstigsten Verbältnissen leicht ausgeschieden werden können.

Wir können daher in der Chloridretention in der Schwangerschaft nur ein Zweckmässigkeitsprincip sehen, vielleicht insofern, als ihre Vermehrung im Blut die Verringerung der Achloride wenigstens theilweise ausgleichen soll. Bekanntlich wird dieses Ziel nicht ganz erreicht, denn der Gefrierpunkt des Sehwangerenblutes liegt höher als in der Norm.

Birnbaum erwähnt noch die von Winckel gefundene abnorm starke NaCl-Ausscheidung in der II. Geburtsperiode, welche auf die starke Nuskelanstrengung, den öfteren Genuss von Wasser und das Fasten der Gebärenden zurückzuführen seien. Dazu muss ich bemerken:

1. Der Genuss von Wasser setzt den Kochsalzgehalt des Urins herab; ich erinnere hier nur an die Arbeiten von Koevesi, Rotschulz, Strauss, Roeder, Steyrer²). Ebenso wirkt Fasten, sofern es sich (was hier gemeint ist) nur um die Vermeidung von Speisen bei gleichzeitiger Wasserzufuhr bandelt.

2. Muskelarbeit setzt die Kochsalzausfuhr durch die Nieren herab [das ist durch die Arbeiten Koranyi's und seiner Schüler zur Genüge bewiesen $\left.\left.{ }^{3}\right)\right]$.

1) Insbesondere: Magn us, Ueber die Veränderungen der Blutzusammensetzung nach Kochsalzinfusion und ihre Beziehung zur Diurese. Arch. f. exp. Path. u. Pharm. 44. S. 68; ferner: Arch. f. exp. Path. u. Pharm. 44. S. 396 u. 45 . S. 210.

2) Siehe Roeder, Berliner klin. Wochenschr. 1903. S. 428 u، Strauss, Zeitschr. f. klin. Med. 47. S. 407.

3) Siehe vornehmlich Korangi, Zeitsehr. f. klin. Med. 33. S. $23 \mathrm{ff}$. 
3. Die Chloridausscheidung ist wäbrend der Geburt durchaus nicht - wie von Winckel seiner Zeit angegeben wurde - erhöht, im Gegentheil: Die Chloridmenge sinkt pro 24 Stunden berechnet - ebenso wie die Harnwasserabscheidung ${ }^{1}$ ) -- während der Geburtsarbeit (entsprechend den Koranyi'schen Resultaten bei Muskelarbeit) von Stunde zu Stunde. Das haben meine zahlreichen Reihenuntersuchungen ${ }^{-}$unzweideutig bewiesen (siehe Zeitschr. f. Geb. 1. Gyn. Bd. 50. S. 413-415, S. 436, 439 , sowie die Uebersichtstabellen S. 419 u. 453). Ich habe auch die Ursachen dieser Erscheinung ausführlich abgehandelt, sodass ich hier nur darauf zu verweisen brauche.

\section{Wie verhält sich die Chloridausscheidnng bei Nephritis gravidarum und Eklampsie?}

Fälle von reinem Hydrops ohne Albuminurie habe ich nicht in das Bereich meiner Untersuchungen gezogen. Wenn Birnbaum an der Hand dreier Fälle, in denen er die 24stündige Harnmenge und den Chloridgehalt bestimmt hat, und bei denen sich die NaCl-Ausscheidung nicht verringert fand, den Schluss zieht, dass eine "Kochsalzretention" nicht vorhanden sein könne (gemeint ist eine Anstaung der Chloride in Folge Insufficienz der Nieren), so kann ich ihm in dieser weitgehenden Schlussfolgerung nicht folgen. Denn, nehmen wir an, es hätten sich Chloride in Folge vorheriger mangelhafter Ausscheidung im Organismus angehäuft, so kann die NaCl-Ausscheidung:

1. erhöht sein (der Körper sucht den Ueberschuss zu entfernen),

2. erniedrigt sein (der Körper kann augenblicklich nicht einmal das normale Quantum ausscheiden),

3. in normaler Höhe liegen (die Niere vermag die normale Ausscheidung nicht zu übersteigen).

Das sind nicht etwa rein theoretische Erwägungen, sondern derartige Verhältnisse sind uns von der Ausscheidung des Stickstoffs durch die nephritische Niere bekannt ${ }^{2}$ ).

Wir brauchen aber diese Fälle von "reinem" Hydrops gar nicht, wenn wir uns die Verhältnisse bei Nephritis betrachtet haben.

Birnbaum hat in einem Fall die 24stündige Chloridmenge bestimmt: $4,8 \mathrm{~g} \mathrm{Cl}(=7,92 \mathrm{~g} \mathrm{NaCl}$ ). Das entspricht also einer starken Verminderung der Kochsalzausscheidung. Dass derartige Perioden verringerter Chloridausscheidung bei Nephritis gravidarum

1) Zangemeister, Dieses Archiv. Bd. 66: S. 415.

2) Vergl. von Noorden, Lehrb. d. Path. d. Stoffw. S. 366. 
vorkommen können, zeigen auch die von $\operatorname{mir}^{\perp}$ ) ausführlich mitgetheilten Fälle. Es liegt aber auch hier keine Insufficienz der Nieren für Chloride vor, sondern die verminderte Chloridausscheidung ist eine Folge der Verringerung der Wasserausscheidung. Wir wissen heute durch zahlreiche Arbeiten, dass bei parenchymatöser Nephritis hauptsächlich die Fähigkeit der Niere leidet, überschüssiges Wasser zu eliminiren; darauf beruht die Prüfung der Nierenfunction durch Verabreichung von Mineralwässern (Roeder, l. c. u. A.). Ich habe in meiner Arbeit gezeigt, dass es bei Nephritis gravidarum und Eklampsie ebenfalls lediglich und in erster Linie die Wasserausscheidung ist, welche Schwierigkeiten macht. Da aber - worauf ich ebenfalls wiederholt hingewiesen habe - die Chloridausscheidung bis zu einem gewissen Grad der Wasserausscheidung parallel geht (die Chloride werden vom Organismus retinirt, um das Wasser isosmotisch zu erhalten; vergl. hierzu die oben citirten Arbeiten von Magnus), so werden - compensatorisch - auch die Chloride zurückgehalten.

Bei der Eklampsie, welche bei dieser Betrachtung als eine besonders schwere Form der Nephritis gravidarum aufgefasst werden muss, geben sich die Verhältnisse noch klarer zu erkennen. Ich verfüge über eine recht grosse Zahl von serienweise durchuntersuchten Eklampsien (Harn und Blut) und kann daher gerade hierüber ein genaues Bild der in Frage stehenden Ausscheidungsverhältnisse entwerfen.

Betrachten wir die auf S. 453 befindliche Tabelle, so sehen wir, dass der Harn sich zu Beginn der Eklampsie (also noch ehe die letztere ihren Einfluss auf die Urinsecretion in ausgeprägtem Maasse geltend gemacht hat) folgendermaassen im Durchschnitt verhält: Die Nichtchloride werden von Seiten der erkrankten Niere genau so gut ausgeschieden wie hei normalen Schwangeren. Die Harnwasserausscheidung ist auf weit über die Hälfte reducirt, die Chloridausscheidung halb so gross als bei Schwangeren, noch kleiner als bei Kreissenden.

Die Harnwasser- und Chlorid verhaltung nimmt im Verlauf der Eklampsie (im Mittel) noch beträchtlich zu, während sie nach den Anfällen wieder zurückgeht. Dieses Verhalten geht auch aus den einzeln angeführten Fällen eclatant hervor; ich verweise hier be-

1) Zeitschr. f. Geb. Bd. 50. S. 440, 444, 452, von denen die letzten zwei nachher Eklampsie bekamen. 
sonders auf den $5^{1} / 2$ Tage hindurch verfolgten Fall 1! Hier wurde während dieser ganzen Dauer beträchtlich zu wenig $\mathrm{NaCl}$ ausgeschieden (Deficit pro die mindestens $3 \mathrm{~g}$ ). Dabei nahmen am 8. IV. die Oedeme ab, die Anfälle hörten auf, das Bewusstsein kehrte zurück, noch ehe auch nur ein Theil des retinirten Kochsalzes wieder durch den Harn ausgeschieden war.

Es kommt also bei Nephritis gravidarum und noch mehr bei Eklampsie zu einer beträchtlichen Chloridretention; jedoch darf diese Verhaltung nicht als an sich schädlich aufgefasst werden. Sie ist keine Folge einer Niereninsufficienz für Salze, da die im Ganzen schwerer ausscheidbaren Achloride sowohl bei Nephritis gravidarum als auch bei Eklampsie (sogar im Mittel aller Fälle und während der ganzen Dauer der Erkrankungen) in unverringerter Menge (sogar vermehrt) zur Abscheidung kommen. Die NaCl-Retention ist die Folge einer reducirten Nierendurchblutung, da sie bedingt wird durch das zeitweilig starke Sinken des Chloridbruchtheils ${ }^{1}$ ) (d. i. ein Steigen des Koranyi'schen Quotienten $\frac{\Delta}{\mathrm{NaCl}}$, dessen Abhängigkeit von der Nierencirculation wir durch Koranyi kennen gelernt haben.

Wahrscheinlich liegen die Verhältnisse so, dass als directc Folge der Herabsetzung der Nierencirculation die Wasserausscheidung, von der wir oben hervorhoben, dass sie bei parenchymatöser und bei Schwangerschaftsnephritis Schwierigkeiten unterliegt, sinkt. Secundär retinirt der Organismus dann die zur Aufrechterhaltung. der Isotonie nöthige Menge $\mathrm{NaCl}$, wie er es in der That bei experimentellen Wasserinfusionen thut (Magnus); hierdurch kann der Urin für eine gewisse Zeit fast kochsalzfrei gemacht werden (Magnus).

Auf die Ursachen der Beeinträchtigung der Nierencireulation bei Schwangeren, Kreissenden, bei Nephritis und Eklampsie will ich hier nicht näher eingehen; sie liegen sehr wahrscheinlich in arteriellen Gefässcontractionen, welche bei Schwangeren und insbesondere bei Kreissenden, in abnorm hohem Grade bei Nephritis gravidarum und Eklampsie unter dem Einfluss einer (wahrscheinlich toxischen) Noxe auftreten. Alle anatomischen und functionellen Veränderungen der Niere, bei Schwangeren, Kreissenden, bei Nephritis und Eklampsie lassen sich auf diese eine Grundlage zurückführen (vergl. dieses Archiv, Bd. 66, S. 445. Zeitschr. f. Geb. u. Gyn., Bd. 50, S. 456-460, 464 u. 465$)$.

1) Vergl. Zangemeister, Zeitschr. f. Geb. Bd. 50. S. 457. 
834 Zangemeistor, Ausscheidung der Chloride in der Schwangersebaft.

Dass in der That die Chloride nicht in abnormer Menge (d. $h$. mohr als zur Erhaltung der Homotonie der Säftemasse nöthig ist) im Organismus der Nephritischen und Eklamptischen aufgestapelt werden, lässt sich an der Hand der Blutanalysen zeigen:

Der mittlere Chloridgehalt des Serums Eklamptischer beträgt $\left.6,36 \mathrm{pM} .(6,10-6,60)^{1}\right)$, bei Schwangeren $6,50 \mathrm{pM} .(6,30$ bis 6,72$)$, bei Kreissenden $\left.6,26 \mathrm{pM} .(6,12-6,44)^{2}\right)$; er ist also durchaus nicht erhöht.

Es kann nach alledem ron einer pathologischell Anhäufung speciell der Chloride im Organismus bei Nephritis und Eklampsie nicht die Rede sein. Absolut genommen enthält der Körper unter diesen Verhältnissen allerdings mehr Chloride als sonst, relativ (im Verhältniss zum vorhandenen Wasser) aber nicht. Die grossen Schwankungen der Chloridausscheidung bei Nephritis und Eklampsie sind nicht als eine Chloridvergiftung, sondern als eine Selbsthülfe, als ein regulatorischer Vorgang anzusehen.

\section{Therapentische Kochsalzentziehung.}

Es muss demnach auf Grund unserer chemischen Kenntnisse behauptet werden, dass eine Entziehung der Chloride zu therapeutischen Zwecken weder bei nephritischen, noch bei anderen Schwangeren diejenige wissenschaftliche Grundlage hat, welche ihr bei Empfehlung des Verfahrens supponirt wurde. Trotzdem kann ich die Möglichkeit einer Einwirkung der Chloridabstinenz auf ödematöse Zustände nicht von der Hand weisen. Wird nämlich dem Organismus dasjenige Salz $(\mathrm{NaCl})$ vorenthalten, welches er bei Wasserretentionen (um solche handelt es sich ja hier, wie oben gezeigt wurde) benöthigt, um dieses überschüssige Wasser isotonisch zu machen (das im Blut und den Geweben vorhandene $\mathrm{NaCl}$ kann er dazu nicht verwenden, da es gerade nur in solcher Menge vorhanden ist, als die Homotonie erfordert), so ist der Körper bestrebt, das Deficit dadurch zu decken, dass er mit dem Harn weniger oder kein $\mathrm{NaCl}$ mehr abgiebt (Versuche von Magnus).

Je weiter die Entziehung geht, um so unvollkommener kann der Organismus den Salzmangel ausgleichen, er verarmt an Salz, seine Säfte werden (molekular) verdünnt. Dieser Zustand wirkt,

1) Zeitschr. f. Geb. 50. S. 396.

2) Zeitschr. f. Geb. 49. S. 98 u. 99. 
wie Magnusi) durch Infusion hypotonischer NaCl-Lösungen gezeigt hat, diuretiseb, nierenreizend.

Es wird daher in der That durch eine NaCl-Entziehung der Wassergehalt des Körpers sinken müssen, Oedeme werden schwinden, aber auf Kosten der Niere! Die letztere muss dabei irritirt werden schon deshalb, weil diejenigen Chloridmengen, welche nöthig sind, um andere schwer lösliche Salze in Lösung zu halten und in den Harn überzuführen, ermangeln. Es kann somit gelegentlich und in Folge der Chloridabstinenz zu einer Verhaltung anderer Stoffe (Urate und anderer N-Körper) kommen. Dass die Niere wirklich durch diese Cur gereizt wird, geht aus der Beobachtung zahlreicher Autoren (auch Cramer's) hervor, dass es im Verlauf der Entzjehungscur zu Albuminurie kommt. Ob man der Niere diese Mehrleistung zumuthen darf, erscheint fraglich. Nur wenn das bestehende Anasarka bedrohlich ist, die Nieren aber relativ gesund sind, würde ich eine solche Cur demnach für vielleicht berechtigt halten. Klinische Versuche müssen hierüber Rechenschaft geben.

Da wir aber durch die Versuche von Magnus wissen, dass auch die Einverleibung hypertonischer ( $\mathrm{NaCl}$ )-Lösungen harntreibend wirkt, und dass gerade durch dieses Verfahren dem Körper Wasser entzogen wird, so könnte man mit vollem Recht auch den Versuch machen, Schwangere mit Hydrops mit grossen $\mathrm{NaCl}-\mathrm{Gaben}$ zu behandeln. Auch hierdurch wird der Hydrops geringer werden, aber auch hier muss es zu einer Nierenreizung kommen.

Ferrannini (Centralbl. f. inn. Med. 1905. No. 1), welcher eine solche Cur bei Nephritis begründet (er verwirft die Entchlorungscur) und empfiehlt, konnte als unmittelbaren Effect in der That eine Zunahme der Albuminurie feststellen.

Ebenso sind die experimentellen Ergebnisse Brandenstein's (Zeitschr. f. klin. Med., Senatorfestschrift) zu erklären, welcher durch Infusion hypertonischer NaCl-Lösungen bei Kaninchen Albuminurie und Schädigung der Nieren hervorrufen konnte. Nicht das $\mathrm{NaCl}$ ist hier das Gift, sondern die künstlich erzeugte Allotonie.

In gleicher Weise, wie reichliche Kochsalzzufuhr, muss Was serentziehung wirken. Das ist für die acute und chronische Nephritis anch nachgewiesen worden (Mohr und Dapper, Deutsch.

1) Arch. f. exp. Path. u. Pharm. Bd. 44. S. 68. 
Arch. f. klin. Med. Bd. 78); bei chronischer Nephritis geht diese Cur aber ebenfalls mit einer Steigerung der Albuminurie einher. Die weitere praktische Erfahrung wird lehren, ob solche diätetischen Maassnahmen zweckmässig sind oder nicht.

Zum Schluss muss ich mich noch gegen einen Abschnitt von Birnbaum (1. c. S. 666) wenden, in welchem er auf die Hydrämie der Schwangeren zu sprechen kommt. Er behauptet, dass die Hydrämie in jüngster Zeit eigentlich kaum noch eine Rolle spiele, dass sie theils mit chlorotischen Zuständen zusammengeworfen würde, theils handele es sich um "Oedeme in Folge von Hydrops ohne Albnminurie“. „Blutuntersuchungen bei unseren Schwangeren mit Hydrops ergaben zwar zuweilen eine geringfügige Leukocytose, sonst aber durchaus normalen Blutbefund." Ich verzichte bier auf eine Kritik dieser allgemeinen Vermuthungen und verweise den Autor auf den Boden exacter diesbezüglicher Untersuchungen, durch welche allein eine derartige Aufgabe zu lösen ist. Ich habe in meiner Arbeit (Zeitschr. f. Geb. n. Gyn. 49. S. 92) die Frage der Hydrämie in der Schwangerschaft eingehend erörtert.

\section{$\mathrm{N}$ a c h trag.}

Versehentlich habe ich die mir wohlbekannte Arbeit Büttner's (dieses Archiv, Bd. 79, H. 2) im Text nicht citirt. Büttner hat in gleichgerichteten Untersuchungen über die Nierenfunction bei Schwangerschaftsniere und Eklampsie" meine Resultate fast bis in alle Einzelheiten bestätigt. 